\title{
Requirements for Productivity in the Academic Environment
}

\author{
Aybars Oruc ${ }^{1}$ \\ ${ }^{1}$ Department of Information Security and Communication Technology, Norwegian University of Science and \\ Technology, Gjøvik, Norway \\ Correspondence: Aybars Oruc, Department of Information Security and Communication Technology, Norwegian \\ University of Science and Technology, Gjøvik, Teknologivegen 22, 2815, Norway. E-mail: aybars.oruc@ntnu.no
}

Received: August 16, 2021

doi:10.5539/hes.v11n4p40
Accepted: September 6, $2021 \quad$ Online Published: October 3, 2021

URL: https://doi.org/10.5539/hes.v11n4p40

\begin{abstract}
Modern life is improving as a result of the research that corporations, research centres, and universities, in particular, conduct. Moreover, besides their teaching function, the quantity and quality of universities' research activities comprise an essential criterion for measuring the university's quality. Today, universities around the world face global competition. Although one facet of the effort to attract productive researchers entails offering more and more, individual incentives are not enough. Universities must also create an attractive academic environment for researchers. This study sought answers to the following question: "What incentives and requirements are necessary to create a productive academic environment?" As the result of a literature review in pursuit of the study aim, the study findings include a total of 10 incentives for researchers and requirements for universities to build a productive research environment in academia.
\end{abstract}

Keywords: higher education, university, researchers, requirements, incentives, productivity, academia

\section{Introduction}

Education is among the most important functions of universities in helping individuals acquire knowledge, skill, attitude, and behaviour (Eneh, 2007). Universities are also vital in the development of the future leaders of a nation. However, a university is not merely a school. In addition to conducting teaching activities, academics carry out research activities at universities, often leading to advances in contemporary science. In particular, universities produce core knowledge. Consequently, other parties may obtain the produced knowledge, shape it to their purposes, and provide it to the public as a product.

The most important determinant of the evolution of human beings is science. Science is divided into three fundamental fields: formal sciences, natural sciences, and social sciences. Investigations in these academic areas are carried out by following an appropriate research method, which may be novel or traditional.

Productivity is represented by the ratio between input and output (Eneh, 2007). International competition in academia forces universities to obtain high-quality research output, which typically takes the form of publications. Such output requires two inputs - productive researchers and a supportive university environment. Notably, poor input results in poor output (Eneh, 2007).

This study is organised as follows. Section 2 describes the results of previous studies. An explanation of the study methodology is presented in Section 3. Section 4 offers various ways of measuring research productivity, followed by a discussion in Section 5 of incentives for researchers and requirements for universities to enable a productive research environment in universities. Section 6 offers the study findings. Finally, Section 7 concludes with a summary and suggests research questions for further potential research.

\section{Related Work}

Fitzenberger and Schulze (2014) conducted a survey among postdocs in Germany. The researchers divided the respondents into three groups, including motivated optimists, confident academics, and frustrated pessimists. Half of the postdocs expressed a strong desire to conduct academic research. According to the postdocs who participated in the survey, the United States offered better opportunities than Germany due to higher salaries, the tenure track system, and better career prospects. In contrast, young researchers in Germany indicated concerns regarding job security.

Meanwhile, Unger et al. (2010) discuss the effect of financial remuneration on biomedical research. The authors 
reported that both researchers and participants receive financial remuneration. Although people are necessary for clinical trials, finding volunteers can pose a challenge. Thus, paying individuals for clinical trials has become a topic of discussion when considering research ethics.

Eneh (2007) focuses on the productivity of Nigerian universities. The author discusses problems related to academic life in Nigeria in terms of university funding, incentives for academics, and the development of facilities as part of a university's infrastructure. Moreover, the article includes suggestions for improving the productivity of Nigerian universities.

Some institutions have attempted to compare teaching and research by assigning arbitrary values to various activities. For example, Yucel and Demir (2018) consider scoring-oriented academic performance, describing how academics in Turkey collect scores based on their work, such as patents, awards, and citations. Among the limitations they note, the researchers identify that 30 points can be collected for each category, with a maximum of 100 points. After enabling the scoring system, academics were not willing to engage in teaching activities compared to research activities.

Research productivity is another area of interest. Fursov et al. (2016) report many findings related to improving productivity, including working conditions, academic mobility, and income. One of the most interesting findings concerns foreign language proficiency. Scholars' ability to read foreign research in the original language makes more publications available to them. Another impressive finding is that older researchers are more productive than their younger colleagues.

\section{Method}

A literature review was conducted to identify potential incentives and requirements that contribute to a productive academic environment. To this end, the study was divided into two parts. First, methods for measuring research productivity were evaluated. Then, elements of the productive research environment consisting of researchers and universities were reviewed. Incentives for productive researchers and requirements for an attractive academic environment were analysed. Several databases, including ScienceDirect, Taylor \& Francis Online, and Springer, were used for the initial scanning. Next, Google Scholar and ResearchGate were used to collect more publications. Notably, although researchers are a key element in the industrial arena as well as universities, this study focused on academia.

\section{Measuring Research Productivity}

Researchers' output can serve as a measure of research productivity in terms of quality and quantity (Ohio State University, 2018). For example, four criteria used for this purpose include Impact Factor / CiteScore, citation number, journal collection, and the number of the publication. Quality is represented in the elements of Impact Factor / CiteScore, number of citations, and quality of the journal collection. Meanwhile, the number of publications reflects quantity.

Evaluating the results of productive researchers can be challenging. One researcher might be a co-author on all the papers that a research group has produced, while another researcher may prefer to write papers alone. One approach is not necessarily more productive than the other. In measuring performance, another critical factor is that researchers' performance should be compared to that of other researchers within the same research field. (MacLeod \& Urquiola, 2021).

\subsection{Impact Factor and CiteScore}

This discussion focuses on two scores that were developed to measure the citation impact of journals. Web of Science offers the Impact Factor. Meanwhile, CiteScore is used by Scopus.

Web of Science's calculation of the Impact Factor of journals comprises two components. First, the Numerator represents the number of citations in the current year for items published in the previous two years. The Denominator, which is the second component, indicates the number of substantive articles and reviews published in the same two years. (Garfield, 2006).

The CiteScore, which is similar to the Impact Factor, also uses a numerator and denominator for its calculation. The main difference is that the Impact Factor is based on the previous two years, while the CiteScore covers a four-year window (Elsevier, n.d.).

The Impact Factor and CiteScore are useful to authors, libraries, and universities. For example, authors may consult these scores when choosing a journal where they wish to submit their studies. Libraries may make collection development decisions based on this quantification. Universities, in their turn, might use these values to measure academic productivity and in their consideration of promotion for academics (Ohio State University, 
2018).

\subsection{Number of Citations}

Citation is a critical measure reflecting the quality of a research endeavour (Ohio State University, 2018). The term refers to earlier research given in a study's bibliography or footnotes indicating the usage of an idea or statement belonging to prior research. Online tools, such as ResearchGate.net or Google Scholar, make it possible to approximate how many times a paper was cited by other researchers. Citation indexing may be traced as far back as the 1950s (Kpolovie \& Onoshagbegbe, 2017).

Researchers' productivity may be measured via different index methods, such as the Hirsch index (h-index), index ten (i10-index), index twenty (i20-index), global citation (g-index), or excess citation (e-index). Google Scholar provides h-index and i10-index information (Kpolovie \& Onoshagbegbe, 2017). The h-index was proposed by Jorge Hirsch (2005). It is easier to explain the h-index with an example: "h-index: 15" means that each one of 15 publications has 15 citations at least. Lastly, the i10 index, first proposed by Google Scholar in 2011, refers to the number of papers with at least 10 citations (Noruzi, 2016).

\subsection{Journal Collection}

Journals can be categorised by quality. For instance, Web of Science (n.d.) lists six core collections, including the Science Citation Index Expanded (SCIE), Social Sciences Citation Index (SSCI), and Emerging Sources Citation Index (ESCI). Meanwhile, Scopus divides journals into four categories by quality: $1^{\text {st }}$ quartile, $2^{\text {nd }}$ quartile, $3^{\text {rd }}$ quartile, and $4^{\text {th }}$ quartile. Specifically, the $1^{\text {st }}$ quartile involves the highest quality journals, while the $4^{\text {th }}$ quartile consists of the lowest quality journals. In addition to Web of Science and Scopus, other databases are available to discern the quality of various journals, for instance, the Norwegian Register for Scientific Journals, Series, and Publishers. Notably, high-quality journals are always peer-reviewed.

\subsection{Number of Publications}

The quantity and quality of publications are both important considerations. Emphasising quantity above all else may result in publishing study findings in low-impact journals (Kyvik \& Aksnes, 2015). That said, competition in the academic environment leads researchers to publish as many articles in high-impact journals as possible (Bruton et al., 2020). Thus, any reward system that is implemented should consider the reputations of the publication channels to avoid a distortion of publication quality (Kyvik \& Aksnes, 2015). The aforementioned index methods, such as h-index or i10-index, could be used to determine the quality of publications. Nevertheless, quantity is crucial in addition to quality for a productive researcher.

\section{Elements of a Productive Academic Environment}

The study established two essential elements that are involved in a productive academic environment: universities and researchers. Each element requires support in different aspects.

\subsection{Researchers}

In this study, Researchers refers to individuals who have academic positions at universities. Institutions of higher education offer a variety of academic positions, such as research fellow, assistant professor, associated professor, and full professor. Research fellows consist of M.Sc.- or Ph.D.-level students and postdocs.

Researchers are a primary factor for high-quality universities. University committees are charged with evaluating researchers in making hiring decisions (Bruton et al., 2020). In particular, researchers who are at the cutting edge of a field should be hired to attain high-quality research. However, hiring productive researchers also involves competition - from industrial sector as well as between universities. As a result, universities face the need to find ways to motivate potential hires and retain productive researchers.

Acquiring productive researchers early in their careers may be an effective approach. Individuals who have obtained a Ph.D. may express a preference for working in academia or industry. After the Ph.D. level, the postdoc is often the first academic position in academia. Encouragement of Ph.D. graduates plays a vital role in keeping them in academia. Increasing age at the postdoc level may lead postdocs to choose to work in an industrial setting rather than academia to reduce personal risks (Chlosta et al., 2010). Therefore, immediately offering a position (e.g. postdoc) to an individual completing a Ph.D. degree is a positive approach to keeping Ph.D. graduates in academia (Enders \& Bornmann, 2002).

In addition to the initial hiring process, university committees have jurisdiction over the promotion process. Thus, the quality of an academic's publications, success in finding research funds, and supervision of doctoral students are indicators for promotion at many UK universities. Research performance, in particular, is a significant factor in promotion to professor (Hare, 2002). 
The quality of a research endeavour depends on the individuals in a research group (Fitzenberger \& Schulze, 2014). Outstanding researchers can positively affect the performance of the group as well as individual researchers, especially junior researchers. This phenomenon, called the "Superstar Effect", was introduced by Sherwin Rosen (1981). In academia, in particular, this effect has been described as follows: "Groups consisting of an exceptionally high-performing member, or superstar, would achieve greater group-level academic performance than groups consisting of members who performed similarly" (Nihalani et al., 2010). Moreover, the superstar effect has an impact on individuals as well as groups.

Several factors, such as age, sex, or marital status, may affect researchers' productivity (Fox, 2005). Moreover, primary employment in a non-academic sector has been shown to negatively affect research productivity (Fursov et al., 2016). In contrast, factors that may improve productiveness include higher salaries, fewer teaching activities, and enhanced laboratory space (MacLeod \& Urquiola, 2021).

\subsection{Universities}

Universities have different functions. Scientific research is one of the most significant activities of a university (Shen \& Tian, 2012). Teaching is also a given for these institutions.

University-based research should promote the founding of new companies. Newly established companies promote competition among a country's existing businesses. Moreover, such industry expansion opens new local job opportunities and can generate a financial return to the university. Lastly, the knowledge resulting from research may be transferred to the industrial sector (Bekkers, 2010).

Some of the currently available university rankings include Webometrics, Times Higher Education (THE), and Center for World University Rankings (CWUR). Although each party may implement different criteria in ranking universities, research activities are undoubtedly a critical factor. In particular, universities seeking a higher position in university rankings must publish a large volume of high-quality research studies.

\subsection{Incentives for Researchers and Requirements for Universities}

Many career job opportunities are available for productive researchers. That's why incentives are necessary to attract them to work at a university. Besides transfers from competing universities, keeping current researchers at the same university is another difficulty for university management. Personal incentives alone are not adequate to hire or keep researchers. The working environment, and possibly the living environment, can affect a researcher's vocational decision-making. Thus, the responsibility of university management is to create, to the greatest possible extent, an attractive environment for researchers.

\subsubsection{Motivation}

Identifying ways to motivate academics to engage in teaching and research activities is necessary. Motivation is a significant element in increasing the number and quality of research investigations. Motivational factors can be divided into two groups: intrinsic and extrinsic (Victor \& Babatunde, 2014). While salary provides one form of incentive for a researcher, it is not the only factor (Victor \& Babatunde, 2014). Different aspects that may appeal to researchers include contributions to science, answering a specific research question, or financial remuneration (Unger et al., 2010). Creative work, social importance, and professional development may also inspire researchers (Fursov et al., 2016). Leadership style is also a critical factor for the motivation of researchers. Solving problems democratically, encouraging creativity, or promoting staff development programmes can energise junior researchers in particular (Victor \& Babatunde, 2014). Researchers also place value on recognition of the university by individuals, industry, or academia, considering that the institution's prestige may lead to better opportunities for individuals' future careers in the long run as well as personal satisfaction in the short term.

\subsubsection{Bonuses and Salaries}

Insufficient income may force researchers to seek out additional earning possibilities, such as participating in commercial projects (Fursov et al., 2016). The pursuit of other activities in the effort to earn more individual income can lead to research time lost. Thus, financial incentives can enhance researchers' productivity and satisfaction at universities (Yücel \& Demir, 2018). One practical example is the performance-oriented payment system, the W-salary system for professors, which has been implemented in Germany since 2005 (Fitzenberger \& Schulze, 2014). A system for negotiating researchers' salaries has been implemented in Norway as well as Germany (Kyvik \& Aksnes, 2015). Universities currently employ various rewards, such as bonuses, additional research funds, and increases in salary, despite the additional cost to the institution that these measures entail (MacLeod \& Urquiola, 2021). 
Various countries have established methods of offering financial incentives. Academics in France can receive a bonus of $€ 6000-€ 15000$ per year for scientific research. In Israel, salaries of academics may increase by 13 per cent following successful research. Academics in China receive a bonus for each paper published in a creditable journal. In Nigeria, academics can also earn bonuses to reward their studies (Yücel \& Demir, 2018).

\subsubsection{Funding for Research Activities}

Funding is significant for research activities and directly affects publication productivity in terms of quality and quantity. This analysis identified both internal and external funding options (Kyvik \& Aksnes, 2015).

A university may obtain funding for the research activities it hosts. In 1982, Dutch universities were required to implement this method. The risk of budgetary reductions caused the quality control of research to be taken more seriously by universities despite the protests of universities opposing the new measure (de Zeeuw \& Kapteyn, 1991).

Many countries, such as Russia, South Korea, Taiwan, China, and Japan, provide financial support to proposed projects (Guskov et al., 2018).

\subsubsection{Working Conditions}

Several factors can affect researchers' working conditions, including job security, benefits, or the length of annual leave. Because research results depend on the creativity of the researcher, scholars should be allowed to manage their schedules according to their personal rhythm (Fursov et al., 2016).

Individual time preferences can have a positive effect on researchers (Chlosta et al., 2010). However, although part-time positions may be offered, this practice might lead to a decrease in the number of publications that researchers create in terms of quality or quantity. Job security (i.e. a permanent academic position) is another factor to attract productive researchers, especially early in their careers. Lastly, universities may offer different benefits to researchers, such as exercise opportunities during working hours, mortgage credit, or health insurance (NTNU, n.d.).

\subsubsection{More Time for Research}

Some researchers may have less time for their potential research because of their teaching responsibilities, administrational jobs, and external activities (Kyvik \& Aksnes, 2015). Less time for research causes inherently fewer or low-quality publications. Accordingly, a sufficient portion of weekly work hours should be dedicated to research activities exclusively. Reduced work hours that are unrelated to research imply positive research productivity.

\subsubsection{Academic Mobility}

Academic mobility, such as joint projects, academic conferences, and temporary positions (e.g. visiting researcher), can encourage increasing productivity among researchers (Fursov et al., 2016). Moreover, mobility may facilitate international collaborations (Fursov et al., 2016). Collaboration can also be carried out with industrial players as well as other universities. On the negative side, academic mobility is costly, including expenses for accommodation, food, transportation, and so on.

Academic collaboration enhances academic productivity. Nevertheless, such collaborations should be conducted with highly productive researchers instead of those who are less productive to encourage personal productivity (Lee \& Bozeman, 2005).

\subsubsection{Academic Culture}

Academic culture is required for high-quality research. This feature involves an individual's spirit, ethics, environment, and outlook. Academic outlook refers to a researcher's viewpoint towards academic activities. Academic ethics refers to all norms and rules that researchers must follow. Academic environment refers to the academic atmosphere and physical condition, such as infrastructure, research equipment, academic databases, and books. Academic spirit denotes opinions and spiritual power arising from the institution's past (Shen \& Tian, 2012).

Academic freedom means the freedom of individuals to study, teach, research, and publish without any obstacles (Tight, 1988). Such freedom applies to academics and students alike (Abdel Latif, 2014) and, in both education and research, is an essential principle in academia (Karran, 2009). This characteristic directly affects researchers' productivity (Fursov et al., 2016). Potential reactions against new perspectives and approaches can prevent researchers from pursuing research in the field (Karran, 2009). For these reasons, academic freedom has a positive effect on university excellence and the productivity of researchers (Karran, 2009). 


\subsubsection{External Evaluation}

As mentioned, universities offer teaching activities in addition to research. One possible option for improving the quality of universities might be an external evaluation of teaching and research. For example, national committees made up of independent experts published reports regarding the field of economics in Dutch universities concerning research in 1985 and teaching activities in 1988 (de Zeeuw \& Kapteyn, 1991). These reports revealed the research outputs and education quality at the universities they described.

\subsubsection{Facility}

Infrastructure is another matter requiring review. Campus facilities, the availability of common equipment and special-purpose equipment, laboratory capabilities, and library opportunities, including subscriptions to research databases, may affect research efforts (Shen \& Tian, 2012). Universities may also establish specific research centres to create a research environment while minimising restrictions? (de Zeeuw \& Kapteyn, 1991). Such a centre can also be used for passing on technology to industry. The university's location is another factor that affects researchers' decision-making. Specifically, researchers' selection criteria include the country and city where the university is situated, along with the distance between home and the campus. A researcher may consider different aspects, such as cultural adaptation, security, freedom, the local legal system, and difficulties in daily life.

\subsubsection{Postdoc or Ph.D. Research Fellows}

Every researcher has the same 24 hours in a day. The time available to senior researchers (e.g. full professors) may be relatively limited for research purposes due to their administrative responsibilities. As one possible solution, the contributions of research fellows may reduce the time that senior researchers need to invest in conducting a research study. Moreover, some research involves interdisciplinary efforts, which research fellows may supply as well. Furthermore, the size of the research group affects research productivity in terms of quality and quantity. Lastly, both a research group and individual researchers can take advantage of the aforementioned Superstar Effect.

\section{Results}

Four criteria were established to measure researchers' productivity: Impact Factor and/or CiteScore, number of citations, journal collection, and number of publications. These factors only relate to research productivity; however, an academic may also be productive in terms of different aspects (e.g. teaching activity, administrative ability), which should be considered. Identifying criteria for evaluating researcher productivity is necessary. For instance, academics might collect scores from different scientific activities, such as projects, exhibitions, patents, awards, and contributions to activities that disseminate knowledge (Yücel \& Demir, 2018).

Different citation index methods are available, such as h-index and i10-index, used by Google Scholar. Additional tools include i20-index, g-index, and e-index.

The study identified 10 incentives and requirements for researchers and universities. These positive measures include motivation, salary and bonus, funding for research activities, working conditions, more time for research, academic mobility, academic culture, external evaluation, facility, and postdoc and Ph.D. research fellowships.

Without a doubt, productivity is an essential property for a researcher. That said, other character qualities, such as leadership, extroversion, openness, creativity, ability to work in a group, and sensitivity towards research ethics are equally significant.

Industry experience on the part of researchers is another benefit. Awareness of the industry-based working parameters can influence the results and success of a research investigation. Guest lecturers from industrial sector can be hired to create a link between the academic and industrial sectors.

Universities should request output from researchers while clearly defining their criteria for success. Therefore, university management should consider the Superstar Effect to enhance mass productivity.

\section{Discussion}

Universities are required to promote advancement in scientific fields in the areas of formal sciences, natural sciences, and social sciences. Any university that aims to be globally competitive must prioritise two strong elements: productive researchers and a supportive environment in academia. This study was carried out to identify potential incentives for researchers and requirements for universities to create a productive research environment in academia. A literature review was conducted to fulfil the research purpose. The study findings include consideration of potential criteria to measure research productivity. Moreover, this investigation identified 10 incentives and requirements related to the topic under investigation. Recommendations for future 
study entail determining the weights of proposed incentives and requirements for productivity in academia.

\section{References}

Abdel Latif, M. M. M. (2014). Academic freedom: Problems in conceptualization and research. Higher Education Research \& Development, 33(2), 399-401. https://doi.org/10.1080/07294360.2014.881766

Bekkers, R. (2010). An evaluation of incentives and policies that affect research institutions' knowledge transfer activities. In 2009 expert group on knowledge transfer: Final report - 30 November 2009. European Commission: Directorate-General for Research. p. 82-111.

Bruton, S. V., Medlin, M., Brown, M., \& Sacco, D. F. (2020). Personal motivations and systemic incentives: Scientists on questionable research practices. Science and Engineering Ethics, 26(3), 1531-1547. https://doi.org/10.1007/s11948-020-00182-9

Chlosta, K., Pull, K., Fiedler, M., \& Welpe, I. M. (2010). Should I stay or should I go? Zeitschrift Für Betriebswirtschaft, 80(11), 1207-1229. https://doi.org/10.1007/s11573-010-0403-1

de Zeeuw, A. J. de, \& Kapteyn, A. J. (1991). Changing incentives for economic research in the Netherlands. European Economic Review, 35, 603-611. https://doi.org/10.1016/0014-2921(91)90162-C

Elsevier. (n.d.). How are citescore metrics used in scopus? - Scopus: Access and use support center. Retrieved from https://service.elsevier.com/app/answers/detail/a_id/14880/supporthub/scopus/\#tracker

Enders, J., \& Bornmann, L. (2002). Übergangsverläufe und statuspassagen nach der promotion. In M. Wingens (Ed.), Bildung und beruf. Ausbildung und berufsstruktureller wandel in der wissensgesellschaft (pp. 159-177). Juventa. Retrieved from https://eprints.soton.ac.uk/353465/

Eneh, O. C. (2007). Improving productivity in Nigerian universities. Nigerian Journal of Development Studies, $6(1), 35-56$.

Fitzenberger, B., \& Schulze, U. (2014). Up or out: Research incentives and career prospects of postdocs in Germany. German Economic Review, 15(2), 287-328. https://doi.org/10.1111/geer.12010

Fox, M. F. (2005). Gender, family characteristics, and publication productivity among scientists. Social Studies of Science, 35(1), 131-150. https://doi.org/10.1177/0306312705046630

Fursov, K., Roshina, Y., \& Balmush, O. (2016). Determinants of research productivity: An individual-level lens. Foresight and STI Governance, 10(20), 44-56. https://doi.org/10.17323/1995-459X.2016.2.44.56

Garfield, E. (2006). The history and meaning of the journal impact factor. JAMA, 295(1), 90-93. https://doi.org/10.1001/jama.295.1.90

Guskov, A. E., Kosyakov, D. V., \& Selivanova, I. V. (2018). Boosting research productivity in top Russian universities: The circumstances of breakthrough. Scientometrics, 117(2), 1053-1080. https://doi.org/10.1007/s11192-018-2890-8

Hare, P. (2002). Why do academics work? Institutions and incentives. mimeo, Herriot-Watt University.

Hirsch, J. E. (2005). An index to quantify an individual's scientific research output. Proceedings of the National Academy of Sciences, 102(46), 16569-16572. https://doi.org/10.1073/pnas.0507655102

Karran, T. (2009). Academic freedom: In justification of a universal ideal. Studies in Higher Education, 34(3), 263-283. https://doi.org/10.1080/03075070802597036

Kpolovie, P., \& Onoshagbegbe, E. S. (2017). Research productivity: h-index and i10-index of academics in Nigerian universities. International Journal of Quantitative and Qualitative Research Methods, 5, 62-123.

Kyvik, S., \& Aksnes, D. W. (2015). Explaining the increase in publication productivity among academic staff: A generational perspective. Studies in Higher Education, 40(8), 1438-1453. https://doi.org/10.1080/03075079.2015.1060711

Lee, S., \& Bozeman, B. (2005). The impact of research collaboration on scientific productivity. Social Studies of Science, 35(5), 673-702. https://doi.org/10.1177/0306312705052359

MacLeod, W. B., \& Urquiola, M. (2021). Why does the United States have the best research universities? Incentives, resources, and virtuous circles. Journal of Economic Perspectives, 35(1), 185-206. https://doi.org/10.1257/jep.35.1.185

Noruzi, A. (2016). Impact factor, h-index, i10-index and i20-index of webology. Webology, 13(1), 1-4. https://doi.org/10.14704/WEB/V16I1/editorial27 
NTNU. (n.d.). Employee benefits. Retrieved from https://www.ntnu.edu/positions/employee-benefits

Ohio State University. (2018). Libguides: Scholarly impact and citation analysis: Journal impact factor: What is it? Retrieved from https://osu.libguides.com/c.php?g=110226\&p=714742

Priya K. Nihalani, Hope E. Wilson, Gregory Thomas, \& Daniel, H. Robinson (2010). What determines high-and low-performing groups? The superstar effect. Journal of Advanced Academics, 21(3), 500-529. https://doi.org/10.1177/1932202X1002100306

Sharon Unger, Lesley Wylie, Shafagh Fallah, Lee Heinrich, \& Karel O'Brien (2010). Motivated by money? The impact of financial incentive for the research team on study recruitment. IRB: Ethics \& Human Research, 32(1), 16-19. http://www.jstor.org/stable/25703688

Shen, X., \& Tian, X. (2012). Academic culture and campus culture of universities. Higher Education Studies, 2(2). https://doi.org/10.5539/hes.v2n2p61

Sherwin Rosen (1981). The economics of superstars. The American Economic Review, 71(5), 845-858. Retrieved from http://www.jstor.org/stable/1803469

Tight, M. (1988). Academic freedom and responsibility (Vol. 4). Open University Press.

Victor, A. A., \& Babatunde, E. G. (2014). Motivation and effective performance of academic staff in higher education (case study of adekunle ajasin university, ondo state, nigeria). Online Submission, 1(2), 157-163.

Web of Science Group. (n.d.). Web of science collections. Retrieved from https://clarivate.com/webofsciencegroup/solutions/webofscience-platform

Yücel, A. G., \& Demir, S. B. (2018). Academic incentive allowance: Scientific productivity, threats, expectations. International Online Journal of Educational Sciences. https://doi.org/10.15345/iojes.2018.01.003

\section{Copyrights}

Copyright for this article is retained by the author(s), with first publication rights granted to the journal.

This is an open-access article distributed under the terms and conditions of the Creative Commons Attribution license (http://creativecommons.org/licenses/by/4.0/). 